Meta

Journal des traducteurs

Translators' Journal

\title{
Traduire, c'est à dire... Phénoménologies d'un concept pluriel
}

\section{Jean-René Ladmiral}

Volume 40, numéro 3, septembre 1995

La traduction, qu'est-ce à dire? Phénoménologies de la traduction

URI : https://id.erudit.org/iderudit/003369ar

DOI : https://doi.org/10.7202/003369ar

Aller au sommaire du numéro

Éditeur(s)

Les Presses de l'Université de Montréal

ISSN

0026-0452 (imprimé)

1492-1421 (numérique)

Découvrir la revue

Citer cet article

Ladmiral, J.-R. (1995). Traduire, c'est à dire... Phénoménologies d'un concept pluriel. Meta, 40(3), 409-420. https://doi.org/10.7202/003369ar

\section{Résumé de l'article}

The author proposes a three-dimensional analysis, which he refers to as "trois phénoménologies distinctes", to discuss the thorny issue of defining translation. First, within the framework of a linguistic or semantic phenomenology, he looks at the different uses of the term "translation". Second, he uses a psychological phenomenology to examine the psycho-linguistic functions. Finally, he resorts to a philosophical phenomenology to study the parameters and the limits when it comes to conceptualizing translation. 


\title{
TRADUIRE, C'EST-À-DIRE... PHÉNOMÉNOLOGIES D'UN CONCEPT PLURIEL
}

JEAN-RENÉ LADMIRAL

Universire Paris-X.Nanterre el ISIT. Paris. France

\begin{abstract}
The author proposes a three-dimensional analysis, which he refers to as "trois phénomenologies distinctes," to discuss the thorny issue of defining iranslation. First, within the framen'ork of a linguistic or semantic phenomenology, he looks at the different uses of the term "rranslation." Second, he uses a psychological phenomenology to examine the psycho-linguistic functions. Finally, he resorts to a philosophical phenomenology to study the parameters and the limits when it comes to conceptualizing translation.
\end{abstract}

Au regretté Georges Mounin.

Paradoxalement, le concept de traduction fait problème, en tant que tel, et à plusieurs égards. Et pourtant il semblerait que tout le monde sache de quoi il retoume. C'est particulièrement vrai dans le contexte d'une revue comme Meta. dont c'est l'objet propre. Il reste qu'il n'est pas inutile d'opérer un retour réflexif, au moins rétrospectif, sur cet objet : et surtout il s'avère que pour les spécialistes aussi, théoriciens-traductologues ou praticiens-traducteurs, il n'est rien moins qu'évident que de savoir exactement ce que c'est que traduire. À cette question qui reste posée, nous nous attacherons à trouver des éléments de réponse dans trois directions différentes, $c^{\prime}$ est-d-dire... dans la direction de trois phénoménologies différentes (d'où le pluriel par lequel commence aussi notre soustitre, en écho à celui du numéro au sein duquel il vient de prendre place).

Toutefois, les limites d'espace et de temps imparties à la présente étude nous ont contraint de lui laisser un caractère programmatique. En sorte que les pages qu'on va lire ne constituent pas tant l'article que nous avions prévu d'écrire qu'une esquisse. l'esquisse de travaux à venir, prolongeant la présente étude : et c'est pour cette même raison que nous serons également amené. à plusieurs reprises, à renvoyer le lecteur à d'autres travaux que nous avons publiés.

\section{- I -}

Tout d'abord, on n'aura pas pu ne pas noter que le mot «traduction" peut prendre une multitude de sens différents. Au point qu'on en est souvent à se demander si on parle de la même chose et de quoi on parle. Qu'il s'agisse de didactique des langues, de linguistique ou de traductologie, cette polysémie du terme engendre un grand désordre terminologique qui ne contribue pas peu à poser les problèmes d'une façon qui tend à les rendre insolubles. Il est permis de se demander parfois si on a bien affaire à un concept, ou si le même signifiant ne renvoie pas là à ce qui serait des homonymes ! D'où nécessité de désambiguïser. C'est pourquoi nous avons pris au sérieux, à la lettre, le libellé même du titre qu'Alexis Nouss a donné au présent numéro de Meta qu'il dirige : la traduction - qu'est-ce d dire?... et c'est y répondre dans la lettre même de notre propre titre en proposant d'abord ce qui pourrait constituer pour ainsi dire une phénoménologie linguistique de ce concept polysémique, c'est-à-dire en rassemblant les éléments d'une phénoménologie sémantique de la traduction.

Meta, XL, 3, 1995 
Dans l'esprit des limites qui viennent d'être indiquées, on devra se contenter, dans un premier temps, d'un catalogue des acceptions que peut prendre le terme. Et encore, un tel catalogue risque-t-il lui-même de n'être pas encore exhaustif... Après avoir écarté d'emblée des emplois purement métaphoriques (comme «telle manifestation est la traduction d'un malaise plus profond" par exemple). aberrants dans le contexte qui nous occupe, on ira donc des acceptions relevant de ce qui serait un sens minimaliste à toutes celles qui relèvent du sens strict - et ce, en passant par tout un dégradé de sens intermédiaires - avant de devoir prendre en compte certaines significations dérivées ou latérales que vient revêtir ce terme, décidément bien polysémique !

0 . Nous distinguerons déjà deux acceptions différentes au sein même du sens minimal qu'on voit prendre à ce terme - si minimal qu'on sera fondé à n'y voir que des emplois abusifs. Ce serait les deux significations de ce que nous appellerons le «degré zéro" sémantique de la traduction.

0.1. C'est ainsi qu'on parlera, à tort sans doute, de "traduction» pour désigner ce qui n'est qu'une équivalence abstraite établie entre deux termes de langues différentes. Ainsi dira-ton que «la traduction» du mot français émotion sera en allemand: Gemütshewegung ou Gemütserregung : alors qu'il n'y a pas, à proprement parler, de «traduction" pour l'espagnol tapas, etc. C'est, à l'évidence, par un abus du langage courant qu'il est convenu d'appeler encore «traduction» ce qui n'est là que l'équivalence abstraite d'une pure et simple equation interlinguistique du genre pain = bred, c'est-à-dire : $A(L x)=B(L y)$; et sans doute vaudrait-il mieux écrire : $A(L x) \cong B(L y)$.

0.2. En revanche, il n’est pas rare d'entendre des linguistes (bien aussi compétents, par ailleurs. que ceux que nous prétendons être) appeler "traduction" toute forme de transfert interlinguistique constituant le noyau minimal sans doute commun aux activités mentales que mettent en ceuvre les diverses configurations de "langues en contact", allant des différents niveaux de «bilinguisme" à la traduction proprement dite. Il y a là certes un moment psycholinguistique qu'on ne peut pas ne pas présupposer en effel : mais il nous apparaît qu'il est trompeur de l'appeler «traduction».

Cela dit, il reste que c'est encore en ce sens minimaliste, excessivement élargi. qu'est employé le terme par des spécialistes de la traduction. Qu'il s'agisse d'analyser les diverses opérations mentales séquentielles qu'est censé réaliser le traducteur, dans une perspective psycholinguistique donc, ou qu'il s'agisse de construire un algorithme enchaînant les opérations du traitement informatique en traduction automatique (voire en traduction assistée par ordinateur), on voit qu'il y a toujours au milieu de la chaîne séquentielle une opération — appelée précisément «traduction" ou «transfert» — qui constitue le moment central où se joue l'essentiel et par rapport auquel les opérations antérieures sont comme autant de phases d'une prélecture démultipliée et les opérations ultérieures font figure de phases d'une postédition analysée en détail'. Il y a là une ambiguité à éviter : mais il y a surtout un problème à résoudre ; et il n'est rien moins que sûr que nous soyons parvenu à aller beaucoup plus loin dans les pages qui suivent...

1. On en viendrait ensuite à ce que nous appellerons le «degré réduit» de la traduction. On entendra par là un travail qui est déjà de la traduction proprement dite, mais qui se situe dans le cadre de l'institution pédagogique, scolaire puis universitaire, el qui fait l'objet. pour cette raison, de tout un ensemble de limitations et de scotomisations. Il s'ensuit les diverses formes et modalités de ce qui constituera, pour ainsi dire, une traduction pédagogiquement réduite.

1.1. Dans le cadre scolaire et, plus généralement, dans toute formation initiale à l'apprentissage des langues vivantes étrangères, on rencontre une forme de "traduction" minimale qui n'est en fait qu'un artefact psychopédagogique : le surcodage mental. Quelle que soit la "méthode» utilisée, quand les apprenants se trouvent confrontés à un 
signifiant inconnu de la langue étrangère qui leur est enseignée (L2), ils tendent spontanément à le sémantiser en l'identifiant à un signe (signifié + signifiant) de leur langue "maternelle" (Ll) : c'est ce qu'il est convenu d'appeler la utraduction spontanée ou "silencieuse». Outre les inconvénients pédagogiques pour l'apprentissage de L2 que cela peut représenter et que ce n'est pas le lieu ici de discuter, il est clair que là, on n'en est pas encore à de la traduction, fût-ce en un sens élargi ; c'est pourquoi on devra plutôt parler de surcodage que de "traduction", quand bien même on y sous-entend les restrictions qu'implique le cadre de l'institution pédagogique ${ }^{2}$. «On entend souvent dire que, même quand le maître ne traduit pas, les élèves 'traduisent' mentalement ${ }^{3}$ : or il est évident que, dans la phrase qui vient d'être citée, le verbe traduire désigne des opérations très différentes.

1.2. Si les apprenants «traduisent» - dans le sens qui vient d'être critiqué parce que l'emploi du terme est en l'occurrence un abus de langage, mais aussi parce qu'il y a là une réaction spontanée novice à leur apprentissage et dont il reviendra à une didactique des langues efficace d'organiser l'inhibition - il arrive aussi que le maître ait à traduire (cette fois-ci sans guillemets), ainsi qu'il vient d'être indiqué. Cette "traduction magistrale", comme l'appelle Henri Besse 4 , a pour fonction de faire comprendre aux apprenants les signifiants de langue inconnue (L2), de les sémantiser, en ayant recours à des équivalents bien choisis dans la langue qu'ils sont censés maîtriser (LI), avec la garantie du maître, à la différence de ceux qu'ils improvisent à l'aveuglette quand ils pratiquent la "traduction" au sens d'un pur et simple surcodage.

C'est aussi mutatis mutandis ce que nous avons appelé la «traduction minimale»'s. c'est-à-dire celle que tolère la méthode directe d'enseignement des langues, en fin de leçon, pour s'assurer qu'au bout du compte le texte en langue étrangère sur lequel on a travaillé a été compris pour l'essentiel, quand même ! Au reste, quelle que puisse être la rigueur méthodologique des orthodoxies didactiques présidant à la mise au point des "méthodes de langues", les enseignants auxquels il revient de la pratiquer, c'est-à-dire de les «appliquer» dans la réalité de la classe, n'ont jamais quant à eux complètement renoncé à ce que nous nous sommes plu à appeler "l'hérésie discrète d'y recourir de façon plus ou moins marginale»o.

1.0. À un autre niveau - plus élémentaire, s’agissant de traduction, mais aussi plus élaboré dans une perspective didactique - le maître pourra avoir recours à diverses procédures pour sémantiser les éléments inconnus de L 2 qu'il introduit dans la progression où il engage ses apprenants : il pourra exhiber des realia référentiels, en donner la représentation figurée sous forme de dessins, en proposer la paraphrase descriptive en L2, voire en Ll, les intégrer dans la simulation d'une mini-dramatisation en classe (là encore en $\mathrm{L} 2$ ou en L1), etc. Il n'est que trop évident que, même dans les cas où il est fait usage de la langue maternelle (L1), on ne saurait proprement parler de "traduction" (d'où le choix de la numérotation que nous avons adoptée pour cet alinéa, et la liberté que nous avons prise de la faire déroger à l'ordre canonique de la progression arithmétique). et il conviendra de parler plutôt de procédures de sémantisation : c'est teliement vrai qu'un des mérites essentiels de ces procédures sera le flou où il reviendra au pédagogue de s'efforcer de les laisser, de sorte que puissent être évités (autant que faire se pourra) les effets de ce qu'on appelle, depuis Larry Selinker, la «fossilisation» des «interlangues». Point n'est besoin de rappeler ici que le travail du traducteur, c'est précisément le contraire $^{7}$ ! Encore une fois, pour le traducteur, «la nuance n'est pas un luxe, elle n'est qu' un aspect de la précision"...

1.3. De ce qui serait l'essentiel en ce qui concerne ce qu'il convient d'appeler proprement de la traduction dans l'institution pédagogique, nous ne dirons ici presque rien, pour plusieurs raisons. D’abord, il y aurait trop à dire : ce serait la matière de toute étude 
sui generis. Et, de fait, de telles études existent déjà en assez grand nombre ; à commencer par nos propres travaux sur la question, déjà cités (en notes ${ }^{8}$ ). Au surplus, dans le contexte de publication où paraît le présent article. c'est un sujet que tous connaissent, ne fút-ce qu'au travers des souvenirs qui leur restent de leur expérience scolaire de la chose ; et que peut-on apprendre à des spécialistes de la traduction sur leur première rencontre avec ce qui esı devenu leur métier, voire leur objet ?

Surtout, dans ce contexte qui est le nôtre, c'est ce que nous avions appelé la «traduction traductionnelle» qui a lieu de retenir notre attention et non pas tant la dite traduction pédagogique dont les deux formes canoniques sont le thème (1.3.1.) et la version (1.3.2.), en vertu de la bizarrerie du vocabulaire français que l'on sait. Au reste, cette asymétrie lexicale peut sembler faire écho ou allusion a l'asymétrie réelle qui existe entre thème et version. Encore une fois, et dans l'esprit de ce que nous avons annoncé comme n'étant qu'un catalogue balisant la polysémie du concept, nous nous contenterons de renvoyer ici à l'étude détaillée que nous avons publiée sur la traduction pédagogique. et plus particulièrement au thème et à la version".

2. À vrai dire, il n'en ira pas tout à fait autrement pour la traduction au sens strict, pour des raisons comparables à celles qui viennent d'être invoquées. C'est a contrario que nous avions défini ladite traduction traductionnelle ${ }^{10}$ par opposition aux modalités de la «traduction pédagogique», celte dernière étant déterminée par les scotomisations institutionnelles qui la définissent (au sens étymologique du mot où c'est dire qu'elles lui fixent des limites, ainsi qu'il a été indiqué). S'il fallait en esquisser une définition positive, on pourrait commencer par dire que la traduction proprement dite, c'est la production d'un véritable texte, existant en lant que tel, en langue-cible ( $T t$ ) pour un public ignorant la langue-source (Lo) dans laquelle était rédigé le texte original (To). Ce serait maintenant le «degré plein" de la traduction : mais là aussi il y aura lieu d'opérer quelques distinctions. Traditionnellement, on opposait traduction littéraire et traduction technique. Les termes étaient ambigus, puisqu'on ne savait pas notamment si c'était opposer là deux catégories proprement traductologiques ou seulement deux étiquetages à caractère socioprofessionnel renvoyant essentiellement à des modes de rémunération très inégaux...

2.1. Surtout, par une synecdoque de commodité, on tendait à subsumer sous ladite traduction "technique» des choses tout à fait différentes. Il s'agit en effet de traduire non seulement des textes techniques (stricto sensu) et scientifiques, ou des textes plus spécifiquement médicaux, par exemple, mais aussi des rapports politico-institutionnels (dans les institutions internationales notamment), voire des textes de lois, etc. C'est pourquoi on a pris I'habitude de regrouper tout cela sous le concept de traduction des textes pragmatiques; mais il nous apparaît que le terme est ambigu, puisqu'il désigne aussi par ailleurs une direction essentielle et encore récente des recherches linguistiques, comme on sait, ainsi qu'un courant traditionnel de la philosophie américaine, sans parler de l'acception que prend le mot dans le langage courant pour ainsi dire en aval de ce dernier sens philosophique. Aussi préférons-nous reprendre, par commodité encore. la désignation de traduction professionnelle - et ce, bien qu'elle ne soit elle-même tout à fait exempte d'ambiguité.

Le paradoxe est qu'en l'espèce, nous savons tous de quoi il s'agit, nous connaissons bien le «référent», mais nous sommes embarrassés pour le choix du signifiant terminologique permettant de l'étiqueter. Quoi qu'il en soit, c'est, quantitativement, le domaine de la traduction le plus important : mais justement, dans le cadre où s'inscrit la présente étude, nous en traiterons par prétérition, laissant à d'autres travaux le soin d'en faire l'analyse. Un autre paradoxe est que plus on approche de l'essentiel, plus on est amené ici à n'y faire que référence, d'une façon quasiment allusive. À vrai dire, le para- 
doxe n'est qu'apparent. dans la mesure où, autrement, il faudrait se payer le ridicule de faire la leçon à des collègues et/ou à des confrères (consœurs) eux-mêmes (elles-mêmes) spécialistes : ou alors, il conviendrait de consacrer à chaque problème évoqué toute une étude sui generis (que nous sommes bien obligé de réserver pour l'avenir...).

2.2. Ainsi serons-nous amené à en user aussi a fortiori pour ce qui mériterait d'être considéré comme cour de la traduction. Là encore, ce qu'on range sous le nom de traduction «littéraire» (lato sensu) constitue un ensemble assez lâche. Là encore, nous préférons à cette synecdoque abusive le recours à une catégorie qui, cette fois-ci, est tout à fait adéquate à son objet: la traduction des auvres - dirons-nous, reprenant à Antoine Berman cette formule heureuse - voilà qui permet d'y subsumer des genres aussi fondamentalement différents que proprement essentiels.

2.2.1. Il y a d'abord la traduction littéraire, qu'en l'occurrence on entendra au sens strict. Il est encore moins nécessaire d'en parler ici que de toutes les autres modalités de traduction que nous avons cru bon de mentionner dans le présent «catalogue», dans la mesure où c'est sans doute le domaine de la traduction qui a suscité le plus grand nombre de publications de toutes sortes, de tous niveaux et de toutes tailles... (concurremment avec, bien sûr, les travaux linguistiques).

2.2.2. Sur la traduction philosophique et la traduction des textes de sciences humaines, nous ne reviendrons pas non plus ici, pour les mêmes raisons que celles qui ont été invoquées plus haut, mais aussi parce que c'est l'un de nos axes de recherche privilégié et que nous y avons consacré un grand nombre de travaux".

2.2.3. Enfin, on ne saurait passer sous silence la traduction des Textes sacrés, ne fût-ce que parce qu'il y a là une ancienneté proprement fondatrice et inaugurale dans l'histoire de la traduction, parce que la Bible reste le livre le plus traduit, etc. Et puis nous nous plaisons à trouver emblématique le fait que justement l'un des pères fondateurs de la traductologie. comme Eugene A. Nida, a mené ses recherches sous l'égide de l'American Bible Sociefy... Ladite traduction des ceuvres religieuses a longtemps moins retenu l'attention que les autres domaines de la traduction - sans doute en raison du positivisme régnant encore naguère sur la linguistique, dont la traductologie n'a été pour un temps qu' un surgeon - mais il est clair que la aussi, la bibliographie est maintenant relativement importante ${ }^{12}$.

Parvenu au terme de ce catalogue, qui est allé des modalités les plus élémentaires et "minimalistes» de la traduction (lato sensu) aux formes les plus achevées de ce qui, pour le coup, mérite pleinement de s'appeler des traductions (stricto sensu), il nous reste à mentionner deux modalités de la traduction qu'on pourra considérer comme «hors champ» dans la mesure où elles sont à part, sinon marginales (d'où leur place dans la présente énumération).

3. En effet, bien sûr, on ne peut pas ne pas faire une place à la traduction automatique (TA) et à la traduction assistée par ordinateur (TAO). Toutefois, c'est bien une place un peu "à part" : à tout le moins en allait-il ainsi jusqu'à présent : de toute évidence, il convenait de traiter séparément de ala traduction humaine» et de «la traduction automatique". Mais les choses sont en train de changer sur ce point : dès lors qu'on a plus ou moins explicitement renoncé à l'ambition initiale qu'une fantasmatique «machine à traduire" pat nous fournir une véritable traduction au sens plein, selon la formule canonique d'une Fully Automatic High Quality Translation (FAHQT), c'est-à-dire notamment depuis que la «traduction automatique» a fait place a la traduction assistée par ordinateur. on voit aussi se multiplier une multitude de "produits" logiciels intermédiaires entre la traduction automatique et la traduction humaine; et la frontière antérieurement établie entre les deux tend à s'estomper. 
Il y a là très certainement une évolution décisive et lourde de conséquences pour l'avenir du métier de traducteur ou. pour parler plus précisément, des métiers de la traduction - un avenir déjà proche, à vrai dire, mais encore difficile à anticiper ${ }^{13}$. Cela n'est pas non plus sans avoir de conséquences au niveau épistémologique, pour autant que nous sommes amené à redéfinir le champ de la traductologie et à repenser le concept de traduction lui-même $(c f$. inf.) : mais, là encore, nous en resterons ici à ces simples indications allusives, en partie parce que nous avons déjà posé ailleurs ces problèmes ${ }^{14}$. Plus généralement, on a affaire là à tout un domaine de recherches foisonnantes. et partiellement secrètes, engagées dans un processus d'évolution et donc d'obsolescence excessivement rapides qui, à l'évidence, ne peut être abordé ici. Soulignons seulement que s'il est vrai, d'un côté, que (comme on l'a vu) la démarcation entre la traduction humaine et la traduction automatique tend à être remise en cause - il reste que, d'un autre côté, si la TA renonce à l'ambition d'achèvement FAHQT, il est permis de se demander si c'est bien encore de traduction (stricto sensu) qu'il s'agit...

4. C'est un peu la même question qu'on peut se poser à propos de ce que certains appellent parfois la traduction orale et qui $n$ 'est rien d'autre, comme on sait, que le travail de l'interprète. Prise au sérieux, l'idée de traduction "orale" tend à rapprocher l'activité de l'interprète de celle du traducteur : c'est une hypothèse de travail plausible. Mais on peut contester, comme le fait un Daniel Gile, qu'il y ait un fondement commun à ces deux types d'activité, ainsi qu'en font le postulat Danica Sélescovitch et Marianne Lederer, et comme nous inclinons nous-même à le penser jusqu'à un certain point ( $c f$. inf.). Quoi qu'il en soit, il faudra là encore introduire des subdivisions.

4.1. Traditionnellement, le travail de l'interprète s'appelait l'interprétariat. Mais, comme on le sait, il a semblé encore assez. récemment aux milieux spécialisés que le terme n'était plus à la mesure de la performance exigée - peut-être parce qu'il rime avec «secrétariat»... Tout au plus conviendrait-il encore aux tâcherons du métier, moins performants, qui ne sont que des interprètes de contact, commis aux tâches subalternes (liaisoninterpreting).

4.2. L'aristocratie du métier que constituent les interprètes de conférences, et dont le corps s'est développé et organisé comme tel sous la pression de l'extension contemporaine des négociations et des organisations internationales, a tenu à changer d'appellation professionnelle : dès lors, l'activité des interprètes de conférence doit s'appeler de l'interprétation. D'emblée, nous avons été porté à voir là une pure et simple «valse des étiquettes" à finalité corporative de promotion professionnelle. en consonance avec la mode contemporaine des euphémisations et du "traitement sémantique» des problèmes... $S$ 'il est vrai que c'est faire écho à «la dimension herméneutique» de toute traduction, "orale» ou écrite, il reste que c'est aussi et d'abord introduire une ambiguitté de plus dans le vocabulaire: et c'est encore plus mal venu en l'occurrence que, justement. celte nouvelle acception du terme côtoie sémantiquement le vrai sens du terme.

4.3. Au-delà de cette querelle de mots un peu byzantine, nous voudrions faire ici une place à une certaine catégorie d'“interprètes» que nous semble exiger l'avenir, et dont il nous apparaît qu'elle ne se situe pas seulement dans une position intermédiaire par rapport aux deux profils professionnels qui viennent d'être évoqués, mais qu'il lui reviendra un rôle tout à fait spécifique. Dès lors qu'on ne s'enferme pas dans le cadre de milieux sociaux et de conjonctures professionnelles restreintes, pour ainsi dire uen cabine», et dès lors qu'on ne s'en tient pas à un rôle de médiation restrictivement linguistique, alors se posent des problèmes beaucoup plus complexes et multidimensionnels de communication interculturelle. Dès lors, il y aurait place pour un créneau professionnel spécifique appelant une redéfinition du travail de l'interprète en fonction des exigences propres aux enjeux nouveaux des sociétés développées multilingues comme l'Europe de 
demain. Aussi avons-nous cru bon de nous attacher à definir le profil professionnel de ceux qui devront être, dans un avenir proche, des animateurs de communication (ou de médiation) interculturelle 15.

4.4. Ce qui, personnellement, nous a mis sur la piste de ce probleme, c'est notamment qu'il nous a été donné de diriger pendant plusieurs années un programme de recherche-action sur les problemes de la communication interculturelle dans un contexte de groupe avec le soutien de l'Office franco-allemand pour la jeunesse (OFAJ). Dans le cadre des groupes bilingues avec lesquels nous avons été amené à travailler - au sens où il s'est agi de ces "groupes» restreints que la psychologie sociale prend pour «objet" c'est toute la dynamique du groupe qui se trouve surdéterminée par les problèmes de «la traduction» tels qu'ils s'y posent, dès lors que tous les membres dudit groupe ne parlent pas la même langue. Mais, là encore, il est bien clair que "la traduction" que réclament certains participants, pour n'être pas exclus de la communication de groupe, a pris dans ce contexte un sens tout à fait particulier - qu'il n'y a pas lieu de thématiser ici, d'autant que nous avons déjà publié les éléments d'un Rapport intermédiaire au terme de la recherche considérée ${ }^{16}$.

Dans les quelques quatre ou cinq configurations qui viennent d'être évoquées, il faut bien dire qu'on n'est plus tant en deçà de la traduction (stricto sensu) que sans doute au-delà. Comme les différentes modalités de la atraduction automatique», les divers profils professionnels ressortissant à la traduction «orale», ou encore «la traduction» qui traverse la dynamique interculturelle des groupes bilingues, toutes ces formes de médiation interlinguistiques se situent un peu en marge du domaine de la traduction proprement dite, pour ainsi dire «hors champ» - d'où leur place, en troisième et en quatrième positions, dans l'ordre de la numération à la Dewey adoptée pour le catalogue de ces acceptions du terme polysémique de traduction. Et c'est ici que nous mettrons un point (provisoirement) final à la phénoménologie sémantique du concept qu a esquissée la première partie de la présente étude en proposant ledit catalogue.

$$
\text { - II - }
$$

0 . Après le catalogue des variations et dérivations sémantiques que connaît le concept de traduction, dont nous nous sommes attaché à dresser bilan au niveau d'une phénoménologie linguistique, il resterait à proposer maintenant les éléments de ce qui constituerait une phénoménologie psychologique du traduire. $|. .$.$| Nous devrons nous con-$ tenter de ne faire qu'indiquer ici trois problématiques, sans les développer.

1. Il resterait d'abord à examiner les quelques fonctions psycholinguistiques qui, pour ainsi dire «en structure profonde», sous-tendent la démarche de traduire pour ainsi dire «en surface», au niveau de ses manifestations (Erscheinung) professionnelles, pédagogiques ou même élémentaires ou spontanées, dont nous avons évoqué la taxinomie sémantique. Cette mise en regard des deux fera l'objet d'une prochaine étude qui ne peut être ici qu'annoncée et que nous nous conterons d'anticiper en évoquant deux (ou trois) exemples très simples correspondant aux deux extrema qui nous semblent baliser le champ de la problématique.

Ainsi les procédures spontanées de sémantisation évoquées plus haut renvoientelles à des modes de fonctionnement psychologiques dont se soutient la fonction "épilinguistique" du langage, dirons-nous en usant d'un terme que nous empruntons à Antoine Culioli en l'infléchissant dans notre sens. Ce n'est que parce que l'interdit méthodologique positiviste, qui naguère encore avait investi la linguistique, jetait l'anathème sur toute forme de «mentalisme» - abandonnant ainsi le domaine de la psycholinguistique aux seuls psychologues - que cette fonction (psychologique) élémentaire a été exclue. 
dans un premier temps, du champ d'études des linguistes et des traductologues, quitte à n'être plus commise qu' aux didacticiens et autres pédagogues.

À l'autre bout de l'échelle, nous sommes tous bien placés pour savoir que la traduction est une fonction sui generis, consciente et organisée, qui fait même l'objet d'un métalangage théorique, (plus ou moins) rationnel (et à la publication duquel est vouée une revue comme Meta...). Entre ces deux pôles extrêmes que constituent la traduction comme fonction métalinguistique et comme fonction élémentaire de sémantisation interlinguistique spontanée, on pourra distinguer différentes fonctions psycholinguistiques de la traduction dont il sera possible de structurer la hiérarchie selon le principe de Jackson - dans un premier temps. et sans préjuger des analyses qui (ainsi qu'il a été dit) seront proposées ultérieurement... L'un des problèmes posés sera de savoir où y situer exactement «la traduction" comme fonction psychosociologique, telle qu'elle vient d'être évoquée dans le cadre de la dynamique des groupes bilingues, d'autant que c'est sans doute plusieurs fonctions psychologiques qui, à proprement parler, sont là mobilisées.

2. Ce serait le lieu de considérer maintenant la problématique des rapports entre traduction et interprétation, auxquels il a été fait allusion plus haut, et de développer l'analyse des fondements psychologiques de ces deux activités : la question essentielle étant de savoir jusqu'à quel point il y a un "noyau» commun au principe de l'une comme de l'autre et/ou dans quelle mesure ce sont deux fonctions psycholinguistiques, deux activités mentales qui s'opposent ( $f$. sup.). Mais, là encore, il nous faudra nous contenter d'en faire mention $[\ldots \mid$.

3. Enfin, et surtout - n'étaient les limites imparties à la présente étude - nous aurions voulu pouvoir y reprendre et prolonger ici la phénoménologie psychologique ou. peut-être plutôt. psychologisante des opérations et activités mentales qui sous-tendent la démarche de traduire. Ce devrait être le lieu où nous avions projeté de développer le schéma des deux phases du fonctionnement psycholinguistique du traducteur et de ce que nous nous sommes plu à appeler le salto mortale de l'interface qui assure l'articulation de la première à la seconde. A vrai dire. nous nous en tiendrons ici à cette simple mention. dans la mesure où il y a là une problématique que nous avons déjà abordée en d’autres lieux ${ }^{17}$ (dans les publications auxquelles nous nous voyons, encore une fois, contraints de renvoyer le lecteur) $)^{18}$.

\section{- III -}

Pour un numéro de Meta qu’à travers son sous-titre Alexis Nouss a voulu situer sous les auspices de la phénoménologie (même si le graphème de pluriel y introduit. presque sournoisement, la liberté de quelques déviations sémantiques), il ne serait pas convenable que fût absente la référence au père fondateur de ce grand courant de la philosophie européenne (ou «continentale», comme disent les anglophones), allemande d'abord, mais aussi française. Ainsi aurions-nous pu (et peut-être dû) commencer en citant Husserl, dont on sait que le geste inaugural de sa philosophie est justement de remettre en cause les pseudo-évidences d'une apparence inaperçue comme telle: ce qu'on traduira, faute de mieux. par "le ça-va-de-soi" - dans la mesure où, tel quel. l'allemand Selbstvertändlichkeit est intraduisible à la rigueur.

Et, de fait, c'est bien ainsi que nous en avons usé avec le concept de traduction en consacrant notre première partie à une phénoménologie linguistique de sa polysémie. La traduction, en effet, "ça va de soi». Tout le monde sait ce que c'est (ou du moins chacun le pense-t-il). Tout le monde a traduit, au moins au collège (et chacun peut. à partir des ruines de ses souvenirs scolaires, se bricoler une «théorie" de la traduction): en cela. la traduction n'est qu'un cas particulier des phénomènes de langage, sur lesquels tout le 
monde a un avis, pour lequel il est prêt à se battre (et il est significatif, à cet égard, que soit si important le courrier reçu par nos journaux au titre de la chronique linguistique que publient certains d'entre eux).

Du coup, ce consensus «évident» (selbstverständlich) sur ce qui nous apparaît être un "point aveugle» de la conscience sociale prend la valeur d'un indice philosophique. Qu'il y ait là un problème, la polysémie du mot traduction en était déjà l'indice, contradictoire, de par ses contradictions mêmes et du fait qu'elle entre en contradiction avec les fausses évidences de ce fameux «bon sens" ou "sens commun" que la tâche du philosophe consiste à interroger. Au reste, bien avant Husserl. c'est la figure tutélaire de la philosophie elle-même que nous eussions pu convoquer ! Socrate est d'abord (dans les "dialogues aporétiques") quelqu'un qui interroge ceux qui savent - qui prétendent ou croient savoir - , quitte à ne faire déboucher que sur la confrontation avec leur propre non-savoir, tel que le manifeste la polysémie du concept sur lequel on s'était interrogé au départ et qui se révèle échapper à la traque intellectuelle qui l'avait pris pour objet. Dans le Ménon. par exemple. l'interrogation sur la vertu n'aboutit qu'à un constat de carence et aux incertitudes de ce que Platon appelle joliment un «essaim de vertus».

Aussi dirons-nous - pour rester dans la même isotopie platonisante - que, s'agissant de la traduction, on a affaire non seulement à un terme polysémique, mais bien plus encore à un concept aporérique. Aussi. après la phénoménologie sémantique (et puis la phénoménologie psychologique), il y aura lieu d'envisager une phénoménologie stricto sensu, c'est-à-dire une phénoménologie philosophique de la traduction ; et, là encore. nous sommes amené à reprendre d'abord certaines des analyses que nous avons déjà commencé à développer ${ }^{19}$.

En effet, compte tenu de tout ce qui vient d'être dit, c'est le concept de traduction lui-même qui fait problème! Si l'on consulte la plupart des diverses définitions qui entreprennent de saisir ce qui fait la nature de la traduction, on en viendra à un énoncé de base du type : la traduction est la pratique qui produit un texte-cible sémantiquement. stylistiquement, poétiquement, rythmiquement, culturellement. pragmatiquement... équivalent au texte-source auquel il vient se substituer : et nous aurions pu encore allonger la liste des adverbes qui viennent ici modaliser l'idée d' «équivalence». De fait, la multiplication des modalités adverbiales ne vient pas ici déterminer le concept. mais en masquer le caractère imprécis. En effet, le concept d'équivalence n'est finalement ici qu'un synonyme de celui de iraduction. En sorte que le type de définition proposé est tout simplement de nature tautologique, c'est-à-dire qu'il nous apprend seulement que la traduction est une traduction!

Si l'on se toume maintenant vers les controverses qui touchent les traductions, on verra qu'assez généralement ceux qui critiquent une traduction le font en reprochant au traducteur de s'être écarté du texte original : comme si la traduction se trouvait inconsciemment définie en termes d'identité. Mais si tel devait être le cas, alors on retomberait dans ce que nous avons critiqué comme étant «l'utopie sourcière de la traduction» 20 et dont la logique impensée est tout simplement que la traduction devrait être la répétition du texte original! Ainsi serait-on ramené a l'immémoriale question du littéralisme en traduction ! et nous devrons renvoyer le lecteur à la polémique qui nous a opposé à des sourciers comme Henri Meschonnic et au regretté Antoine Berman21 : mais il est clair que la totalite des traducteurs professionnels sont ciblistes par construction.

La traduction, cette activité si répandue que nous avons tous pratiquée, ne fat-ce qu'au collège, et qui ne nous faisait pas problème, voilà que nous sommes maintenant en difficulté pour la définir ${ }^{22}$. Ne semblerait-il pas qu'elle soit un indéfinissable ? à la maniżre de ces termes premiers que ne udéfinissent" pas les axiomes d'une théorie «hypothético-déductive» comme la géométrie - et, comme on sait, on dira maintenant 
plutôt, justement : une géomélrie - mais que se contentent de mettre en đuvre les «fonctions propositionnelles» qui en sont déduites. Ce qui revient à "définir" par l'usage (et on n'est pas très loin d'une définition du genre "ça sert à quoi ?"). Il y a là quelque chose d'inattendu et de tout à fait paradoxal, mais dont il convient de prendre acte...

Dès lors, il ne reste plus qu’à se tourner vers des «définitions» qui renoncent à saisir l'essence de la traduction (pour aller encore dans le sens des premiers dialogues, aporétiques, de Platon) en compréhension ; et on devra se résoudre à avoir recours à des définitions réelles: qui désignent leur objet en extension. C'est ce que fait un Gideon Toury, qui définit la traduction comme un "phénomène empirique", c’est-à-dire qu“à l'en croire : est une traduction ce qui se donne pour telle et est acceptée comme telle dans une culture donnée à une époque donnée ${ }^{23}$. Mais, au bout du compte, $n$ 'est-on pas là encore bien près d'une tautologie? Certes, ce peut être une façon de se mettre en devoir de satisfaire à la nécessilé discursive à laquelle est soumis un universitaire de "définir" les termes qu'il utilise. Mais, en fait, on aura besoin d'une autre définition. Aussi ne nous restera-t-il plus qu'à en revenir à la (quatrième) solution minimaliste d'une définition du type : Ça sert à quoi ? justement, comme en usent les enfants... C'est ce que nous avons cru devoir faire d'emblée. dans notre premier livre: Qu'est-ce qu'une traduction ? Ça sert à «nous dispenser de la lecture du texte original» 24 .

Les quelques remarques qui sont venues nourrir notre troisième partie, consacrée à une "phénoménologie philosophique" - qui, dans le contexte de la présente publication. ne peut manquer de sembler prendre l'apparence d'un libellé ad hoc - $s$ 'inscrivent dans le cadre plus général du prolongement de la traductologie que nous nous sommes plu à appeler cum grano salis notre "traductosophie»25. Elles n'en constituent bien évidemment qu'un élément de départ. Il y a là en effet tout un champ de réflexion ${ }^{26}$; car il s'avère que la traduction revêt la fonction d'un dispositif critique inattendu qui, entre autres choses, met en question( $s$ ) le rapport à l'écrit qui est au fondement même de nos sociétés et de notre culture, de notre identité elle-même, à travers notamment la mise en cause du statut de ce qui fait un texte 27 , etc.

Mais sans préjuger des conséquences à approfondir et des différentes extrapolations philosophiques qu'il y aurait lieu de dégager, au-delà d'une phénoménologie philosophique de la traduction. $|. .$.$| , nous voudrions conclure la présente étude par une remarque$ inaugurale de Georges Mounin qui. pour être en quelque sorte l'alpha de sa théorie de la traduction. n'en est pas l'oméga, ni encore moins celui de notre dite traductosophie : "Tous les arguments contre la traduction se résument en un seul: elle n'est pas l'original»2x. Ce qui pourrait bien faire là figure de truisme indique au contraire, répétons-le, la direction d'une véritable réflexion théorique sur la traduction, dont nous venons d'indiquer combien pouvaient être aporétiques les efforts pour la définir et de montrer comment l'assimilation du concept à celui d'identité était un impensé de la traduction - paradoxalement, les pages qu'on vient de lire débouchent sur un aveu d'impuissance qui prend pour nous une valeur authentiquement philosophique : nous dirons que la traduction est un concept premier, un peu comme il existe des «nombres premiers"... 
Notes

1. C'est un point que nous abordons, au passage, dans le cadre de notre contribution aux Actes du colloque de Mons (25-27 avril 1991) : «Lordinateur est une vraie machine à écrire", L environnement raductionnel. La station de travail du iraducteur de l'an 2001. A. Clas et H. Safar (dir.). Montreal, Presses de I'Universite du Québec et AUPELF-UREF, 1992. Sur ces questions, of inf.

2. Sur le contexte pedagogique général de la notion. if. Henri Besse. wDes usages de la langue matemelle dans l'enseignement/apprentissage des langues secondes ou étrangères". Actas del Primer Coloquio Internactonal de Traductologia (2, 3.4 de mayo de 1989). B. Lepinette. M. A. Olivares Pardo, E. Sopeña Balordi (dir.), Universitat de Valencia, 1991, (Quadems de Filologia), pp. 15-20.

3. Ibid., p. 16.

4. Ibid., p. 15 .

5. Traduire: theoremes pour la traduction. rédition revue et augmenté d'une nouvelle préface. Paris. Gallimard, 1994. coll. "Telm. n"246, p. 29 el passim. La première édition de ce livre était parue en 1979. dans la Petite Bibliothèque Payot ( $n^{\prime \prime} 366$ ) : cette seconde édition en est la reprise, augmentée d'une preface (pp. V-XXI), avec une pagination identique (pour le corps du lexte) et quelques cocyuilles en moins. (Nous ferons désormais référence à ce livre en l'appelant, par apocope, nos Théorèmes...).

6. "Pour la traduction dans l'enseignement des langues: 'version' moderne des Humanités”, Les langues modernes, n" 1/1987. numéro spécial : Tradure/lanxue masernelle/langue stranxére. p. 11.

$\therefore$ aTraduction el ambiguité. BRISES. Bulletin de recherche sur l'information en sciences economiques, humaines et sociales, $n^{\prime \prime} 7$. octobre 1985, pp. 59-61.

6. Cf. sup., notes 5 et 6 .

9. "La traduction dans l'institution pédagogique", Langages, $n$ " 28, décembre 1972, pp. 8-39; c'est à cette étude, remaniée et développé. que nous avons consacré tout le deuxième chapitre de nos Théoremes.... op.cit. pp. 23-8.3. Entre-temps, et pour ainsi dire en aval de notre travail. il est paru sur le mème sujet diverses études, et notamment: Elisabeth Lavault. Fonctions de la truduction en didactique des langues. Apprendre une langue en apprenant à traduire. Paris. Didier Erudition. 1986. coll. *Traductologie". n" 2.

11. Theordmes... op.cul. p. 41 squ.

11. Citons seulement nos “Eléments de traduction philosophique", Jean-René Ladmiral et Henri Meschonnic (dir.), La traduction: numéro special thématique de la revue Langue franģaise, n' 51. septembre 1981. pp. 19-34 : et signalons au passage, dans l'esprit classificateur qui est celui des pages qu'on vient de lire. que nous proposions dans cette étude une rypologte de la traduction à trois termes. opposant traduction "technique", traduction littéraire et atraduction philosophique" : c'étail au reste l'occasion de proposer une réception critique, en français. des travaux qu'a publiés Katharina Reiss en langue allemande. Pour le reste. nous renvoyons au quatrième volume de notre Thèse d'Habilitation à diriger des recherches -- récemment soutenue, sous le titre la raductologie' a la linxuistique d la philosophic. le 21 janvier 1995 a l'Université de Paris-X-Nanterre, sous la direction de Michel Arrivé et sous la présidence de Paul Ricorur - intitule Pour une philosophté de la raducrion (338 p.). a paraître.

12. Mentionnons seulement au passage le numéro thematique sur la traduction des textes sacrés : le domaine hiblique, qu'a dirige Alexis Nouss, de la revue $T T R, \mathrm{n}^{\prime} 2 / 199$ ) (au sein duquel nous avons nous-même publié une étude intitulee «Pour une méthodologie de la traduction", pp. 121-138).

13. Cette échéance comporte bien sâr une sérieuse responsabilité pour les formateurs - defi que s'attache a relever un Daniel Gouadec : «Traduction et informatique : les implications pour la formation», Langages. n"116. décembre 1994, pp. 59-74.

14. Cf. notre préface a la rédition de nos Théoremes, op.cit.. p. IX spp., et notre étude sur "Le traducteur et l'ordinateur", Langages, n" 116 , décembre 1994, pp. 5 sqq.

15. Jean-Rene Ladmiral, "Traducción y comunicación intercultural», Acras del / Simposio internacional de traducción. Universidad de Las Palmas de Gran Canaria, 29 enero-l febrero de 1990 (à paraître). C'est au demeurant un probleme dont il nous avait semblé. au colloque de Mons. qu une Karla Dejean Le Féal $n$ 'était pas loin d'avoir soupçonné ; mass, en dehors de quelques formules occasionnelles. nous $n$ 'en avons pas retrouvé la trace dans sa contribution aux Actes cités en note 1.

16. Cf. Jean-René Ladmiral et Edmond Marc Lipiansky, La communication interculturelle, Paris, Armand Colin, 1989, rééd. 1991 et 1995 (Bibliotheque européenne des sciences de l'éducation) : et c'est à une psychologie sociale de la traduction que nous avons consacre toute notre premiere partie, pp. 21-76. Nous allons prochainement entamer un nouveau cycle de ce meme programme de recherche. sur le thème «Traduire les langues, traduire les cultures* (qui donnera lieu lui-méme à publications, à échéance de quelque deux ou trois ans).

17 A Mons et a Strasbourg notamment : c'est un probleme que nous nous sommes trouve paradoxalement amené à traiter dans notre étude déjà citée (cf. note 1): voir aussi «Que le traducteur est un réecrivain", Actes de la journée d'éfudes internationales (Ré)Étritures liftéraires ef utilitaires, organisée par Gertrude Gréciano a I'Université des sciences humaines de Strasbourg 12 mai 19941, à paraître dans Nouveaux Cahiers d'Allemand (publies près de l'Universite de Nancy). 
18. Plus généralement - parvenue au terme de cette $2^{\mathrm{c}}$ partie de notre étude. consacrée à ce qui n'aura été finalement que le plan d'une phénoménologie psychologique de la traduction. nous renvoyons aussi au chapitre V (“Pour une psychologie de la traduction») du premier volume de notre Habilitation cité plus haut. à paraître.

19. Cf. notre préface a la réédition de nos Théoremes... op. cit., p. XVIIl sq

20. "La traduction proligere? - - Sur le statut des textes qu on traduit", Mefa, 35-1, mars 1990, p. 113.

21. Voir notre étude intitulée "Sourciers et ciblistes". Re'vue d'esthétique, n" 12, 1986, pp. 33-42.

22. Un peu comme, a en croire un autre phénoménologue, disciple infidele de Husserl, nous le sommes (in Verlexenhe'it gekommen) quand nous nous posons ala question de l'Etre" (die frage nach dem Sinn vom Sein) : Heidegger, Sein ud Zo's. Tübingen, Nimeyer, 1963. pp. 1 sqq.

23. Gideon Toury. "Translated Literature: System. Norm. Performance: Towards a TT-Oriented Approach to Literary Translation”. In Search of a Theorv of Translation. Tel Aviv. The Porter Institute for Poetics and Semiotics. 1980, pp. 35-50

24. Theoreme of op cit., p. 15

25. "La traduction proligere? - Sur le statut des textes qu'on traduit", loc. cis., p. 15.

26. Ce devrait être, du reste, le thème d'une prochaine livraison de Metu...

27. "La traduction proligère? - Sur le statut des textes qu on traduit». Iox. cir

28. Le's belles infidleles, Paris, Cahiers du Sud, 1955. p. 7 (rééd. aux Presses Universitaires de Lille. en 1994. dans la collection aÉtude sur la traduction»). Que cette citation nous soit une façon de rendre discrètement hommage au regretté Cieorges Mounin.. 